\title{
INFORMATION AND COMMUNICATIONS TECHNOLOGY (ICT) AS THE ENGINE OF INNOVATION IN THE CO-EVOLUTION MECHANISM
}

\author{
Muhammad Suryanegara $^{1 *}$, Ruki Harwahyu ${ }^{1}$, Muhamad Asvial $^{1}$, Eko Adhi Setiawan ${ }^{1}$, \\ Eny Kusrini ${ }^{2}$ \\ ${ }^{1}$ Department of Electrical Engineering, Faculty of Engineering, Universitas Indonesia, Kampus UI \\ Depok, Depok 16424, Indonesia \\ ${ }^{2}$ Department of Chemical Engineering, Faculty of Engineering, Universitas Indonesia, Kampus UI \\ Depok, Depok 16424, Indonesia
}

Shortly after the invention of the steam engine, which revolutionized industry at the end of the nineteenth century, technology was perceived as having only two possible roles: technology was created to comply with market demand or was generated to create markets. These two roles mark the first- and second-generation innovation models that changed the world drastically in the twentieth century. Along with technological advancement in various fields, technology now no longer has the option of two such distinct roles, but rather it exists as a function of the coevolution between technology and ecosystems outside the technology itself. Technology continues to develop as an interplay of these relations. The most obvious example is the rapid development of Information Communication Technology (ICT), which began with the invention of technology to deliver voice communication (thanks to Alexander Graham Bell's telephone). ICT has now been transformed into an "any technological platform," delivering any information over sort of communications technology. The crucial point of the development of ICT was the emergence of the Internet in the late 1970s. Initially, the Internet platform emerged as a military necessity to fragment data communication patterns so that the potential damage to one communication line did not damage the entire communication system. But the ecosystem turned out to require more than just that motivation. The Internet was adopted by non-military industries, educational institutions, and the household. On the basis of this adoption, the ecosystem required continuous development until Internet technology became what we experience today. The Internet has become a prerequisite for the formation of quality people with an orientation toward improving the quality of the human economy.

The development of ICT is what ultimately leads us to the industrial revolution era 4.0, which we face today. With possibilities offered by the sophistication of ICT technology (especially the utilization of the Internet platform), the efficiency of industrial processes becomes a necessity pursued by every business entity. ICT technology is no longer regarded as an enabler of economic activity, but rather it is becoming increasingly specific, enabling industrial process efficiency and strengthening the competitiveness of products, which, in turn, eventually lead to the empowerment of society.

Like a co-evolution process, the need for sophisticated ICT technology continues to grow, creating the need for platforms to support process efficiency and productivity, such as sensor platforms, Artificial Intelligence, machine learning, and robotics automation. Regardless of the technology developed, the industrial revolution era 4.0 has led to a desire to create new innovations that provide economic benefits to the community. Yet, such a technological perspective is applied not only for the ICT-related theme, but also the non-ICT one.

\footnotetext{
*Corresponding author's email: m.suryanegara@ui.ac.id, Tel. +62-21-7270078, Fax. +62-21-7270077 Permalink/DOI: https://doi.org/10.14716/ijtech.v10i7.3777
} 
Of course, the industrial revolution era 4.0 presents many challenges in all areas of life. Drastic changes in technology have a direct impact not only on economic but also on social and cultural life aspects. Both cultural and social changes produce positive and negative impacts. The whole change has the same two-pronged pattern, and ICT has played a prominent role causing such changes. However, remembering its definition, technology is simply a tool to help humans solve their life problems. In this vein, we also return to the proper ICT paradigm, which is that any person should utilize ICT to handle life complexities, while, in parallel, ICT should act as the engine of innovation.

In this context, this edition presents fifteen papers dedicated to promoting ICT research that directly and indirectly contributes to innovation creation in the industrial era 4.0. As this edition consists of 20 papers, the remainder ones have a comparable non-ICT theme which exhibits their distinctive role in the co-evolution mechanism along with the ICT evolvement. They are also promoting how the ICT and non-ICT theme have worked closely in scientific research activities. At the end, the intense research works of these two themes aim to shape technological innovation and competitiveness.

The first paper, written by C.C. Saar, M. Klufallah, S. Kuppusam, A. Yusof, L.C. Shien, and W.S. Han, examines the credibility of Building Information Modeling integration in Augmented Reality (AR-BIM) in the construction industry. The paper first highlights the limited interaction between real and virtual/digital worlds in the construction industry. It then elaborates the proof-of-concept of interoperability between BIM and AR. The discussion presented in this paper aims to raise awareness among construction stakeholders regarding technology adoption.

The second paper, written by S.C. Poh, Y.F. Tan, S.N. Cheong, C.P. Ooi, and W.H. Tan, presents an alternative and simple method for detecting anomalies in the patterns of elderly people's daily home activities. The anomaly may be caused by a health condition, which can then be escalated to produce alert. It is important as the elderly population continues to grow and more young adults are unable to take care of them due to work commitments. The experiment results demonstrated that the model achieved a test accuracy of $90.79 \%$ on a public dataset.

The third paper, written by S. Krishnan, V. Chinthakunta, and S.K. Swee, proposed a Smart Meter Circuit Breaker (SMCB), a shock or fault protection system for a single-phase home electrical system. The system can be used with an off-the-shelf Circuit Breaker (CB) and colonized Authentication Outlet (AO). The system incorporates a novel Restricted Demand Management, which could be used together with the existing Scheduler Demand Management system. The proposed system is able to reduce electricity bills and restricts some hazard-risk appliances from being used at other outlets.

The fourth paper, written by A. Sherif, S. Sherif, C.P. Ooi, and W.H Tan, summarizes the work done in developing the LoRa driven home security system for retirement residential communities, which incorporates hardware and software solutions to provide emergency alerts both manually or automatically. LoRa wireless communication technology is used to connect the devices with the central server. A mobile application provides a chatroom between neighbours, and a web application provides management by the administrator, accordingly.

The fifth paper, written by K. Kalarat and P. Koomhin, introduces a real-time interaction with volume rendering using one-dimensional transfer functions via Virtual Reality (VR) technology. The result is visualized in a stereoscopic 60-fps video using a GPU-based RayCasting shader to produce appropriate levels of light, darkness, and color. The evaluation is conducted with 20 participants, and the survey results show the average usability score of the system is 87.54 , which is a very high score for the application. 
The sixth paper, written by D.C.K. Kho, M.F.A. Fauzi, and S.L. Lim, discusses the use of a fast reciprocal square root (FRSR) algorithm for hardware-based Sobel edge detection. The solution discussed in this paper is proposed to address the ever-increasing demand for high-speed imaging and communication applications. The algorithm is designed directly on hardware of a Field Programmable Gate Array (FPGA). Thus, the speed of the algorithm depends more on the clock frequency, and the logic cell propagation delay usually measures in nanoseconds. Therefore, the speed requirement can be served.

The seventh paper, written by P. Boonbrahm, C. Kaewrat, and S. Boonbrahm, studies the validity of replacing traditional cardiopulmonary resuscitation (CPR) training with an Augmented Reality (AR) system. CPR is an important lifesaving technique used especially to address heartbeat or breathing problems whose fatality reaches $90 \%$ when proper CPR is not performed. The system is implemented using the Unity 3D game engine and Qualcomm's Vuforia software development kit (SDK). A marker-based technique is adopted to present a realistic look at the patient, as well as to indicate the position of the hand on the chest, the number of chest compression per minute, and the pressure that he or she put on the chest. The experiment results confirm that the AR-based training both increase the speed of CPR training and produce more accuracy.

The eighth paper, written by R. Banaeeyan, H.A. Karim, H. Lye, M.F.A. Fauzi, S. Mansor, and J. See, improves pornography recognition using audio features. This approach is particularly important in cases where the visual features are not adequately informative (e.g. cluttered scenes, dark scenes, scenes with a covered body, etc.). It proposes a mechanism that extracts two types of features - pitch and Mel-Frequency Cepstrum Coefficients (MFCC) - to train five variations of $\mathrm{k}$-nearest neighbor $(\mathrm{KNN})$ supervised classification models. The experimental demonstrates an accuracy of $88.40 \%$, F-score of $85.20 \%$, and AUC of $95 \%$ in the task of pornography recognition.

The ninth paper, written by M. Elsayed, M.T. Soe, W.W. Kit, and H. Abdalla, investigates the development of a mobile robot that uses ultrasonic sensors to measure distance and draw a 3D map of its surroundings. The robot is designed to be robust within various obstacles and perform within a narrow space. The results obtained from extensive work on developing the vehicle, as well as testing of the control and 3D mapping algorithm, is presented in this paper.

The tenth paper, written by J.R. Ng, V.T. Goh, T.T.V. Yap, and M.K. Shuib, proposes a reliable transport layer protocol that uses a combination of redundant packets and probabilistic transmission to ensure the reliable transmission of data while maintaining the network's efficiency. The proposed protocol does not require acknowledgement signaling. The results demonstrate that the proposed protocol achieves a $7 \%$ improvement in reliability while maintaining efficiency at $77 \%$ when compared to UDP.

The eleventh paper, written by T.H. Dahawi, Z. Yusoff, R.Q. Shaddad, M.S. Salleh, and J.M. Senior, presents a converged fiber-wireless (FiWi) network architecture, including an OFDMPON and RoF system. Two multiple-input multiple-output (MIMO-RoF) channels are inserted into the left and right sides of the OFDM-PON spectrum using a single-sideband frequency translation (SSB-FT) technique. The evaluation is performed by simulating the proposed FiWi network on a downstream link over a $20 \mathrm{~km}$ standard-PON. The simulation results verify that the proposed network could be a promising candidate for a converged wired and wireless network.

The twelfth paper, written by V. Tilwari, M.N. Hindia, K. Dimyati, F. Qamar, and M.S.A. Talip, proposes a Contention-Window-and-Residual-Battery-Aware Multipath (CRAM) routing scheme. It is proposed for Mobile Ad-hoc Networks (MANETs), where limited energy resources and traffic congestion at the nodes are the crucial issues and may degrade the routing 
performance and Quality of Service (QoS). The scheme embodies a Multi-Criteria Decision Making (MCDM) technique to identify an intermediate node in the optimal path. The evaluation yields that the CRAM routing scheme enhances the throughput, decreases packet loss, and reduces energy consumption compared to the conventional Multipath Optimized Link State Routing Protocol (MP-OLSR) routing scheme.

The thirteenth paper, written by S.E. Lim, R. Ismail, W. Hashim, and A. Baharum, presents the evaluation results of three formulas for capturing vegetation data obtained by an unmanned aerial vehicle (UAV) using the visible light spectrum. The formulas are identified as the Visible Atmospherically Resistant Index (VARI), Green Leaf Index (GLI), and Visible Atmospherically Resistant Indices Green (VIgreen). The detection accuracy results are presented in this paper.

The fourteenth paper, written by T-S. Wong, G-Y. Chan, and F-F. Chua, proposes a Scaling and Fault Tolerance (SFT) algorithm to deploy a preventive or remedial measure based on 16 decision rules for QoS violation detection and prediction. This algorithm is important and useful for cloud service providers, helping them to fulfil QoS as defined in Service Level Agreements (SLA). The evaluation is performed in a cloud simulator with four scenarios to measure its effectiveness in handling a faulty virtual machine (VM) and over- and underprovisioned resources. The results show that the proposed SFT algorithm performs $90-100 \%$ effectively in providing a preventive or remedial measure and reducing the number of VMs when it is not needed.

The fifteenth paper, written by M.A. Summakieh, C.K. Tan, A. El-Saleh, and T.C. Chuah, investigates the use of the Particle Swarm Optimization (PSO) algorithm to perform a dynamic user association by finding the optimal bias values. This issue is important in heterogeneous networks (HetNets) since the use of existing user association schemes may lead to load and power-consumption imbalances among different base stations, which, in turn, may degrade network performance. The simulation results demonstrate that the proposed scheme achieves better load balancing performance compared to a baseline scheme.

The sixteenth paper, written by M.A. Berawi, P. Leviakangas, F. Muhammad, M. Sari, Gunawan, Y.A. Yatmo, and M. Suryanegara explores several models for decision-making in disaster management, especially in emergency resource allocations. These models are required for search and rescue personnel to operate efficiently. However, in Indonesia, the use of technology to help in making decisions in the response phase has not been generally implemented, and decisions are still made subjectively. This paper provides a decision-making model that helps search and rescue teams determine the numbers of personnel to deploy. Therefore, it streamlines the allocation of personnel in a search area and determines the number of personnel based on the area, population density, equipment, and the number of high buildings. The data and knowledge used for reference for this model are obtained from experts in disaster management and experienced practitioners in the field of search and rescue. Fuzzy logic and decision trees can be used to determine the number of victims trapped during an earthquake. Furthermore, they can be used to accelerate the planning phase for search and rescue operations.

The seventeenth paper, written by F. Yulia, V.J. Utami, Nasruddin, and A. Zulys, examines the usage of MIL-101 as the metal-organic framework (MOF) to minimize the $\mathrm{CO}_{2}$ levels in the environment. This paper studies the synthesis, characterization, and adsorption isotherms of MIL-101. The synthesis is carried out via a fluorine-free hydrothermal reaction method. The porous properties, structure, morphology, thermal stability, and chemical functionalities of MIL-101 Cr were measured by the adsorption/desorption of $\mathrm{N}_{2}, \mathrm{X}$-ray diffraction (XRD), scanning electron microscope (SEM), thermogravimetric analysis (TGA), and Fourier 
Transform Infrared Spectroscopy (FTIR) analysis, respectively. The volumetric uptakes of $\mathrm{CO}_{2}$ were experimentally measured at the temperature of 298-308 K and pressure up to $600 \mathrm{kPa}$. The experimental result was correlated with the Toth isotherm model, showing the heterogeneity of adsorbent. The heat of adsorption of MIL-101 was also analyzed, determined from the measured isotherm data, which indicated the strength between the adsorbent and adsorbate molecule.

The eighteenth paper, written by K.C. Chin, L.K. Leong, S-Y. Lu, D-H. Tsai, and S. Sethupathi, investigates a series of varying molar ratios of the NiCe MOF-derived catalyst, which were grown on alumina in one step. This process was carried out in accordance with the reported solvothermal method for the syntheses of $\mathrm{NH}_{2}-\mathrm{MIL}-88 \mathrm{~B}$ with slight modification, followed by reduction to $1023 \mathrm{~K}$ in a $\mathrm{H}_{2}$ environment for one hour. The physical and chemical properties of the catalysts were characterized by XRD, SEM, EDX, ICP and H2-TPR. XRD showed a diffraction pattern that was in agreement with the diffraction pattern of MOF synthesized in previous work, and thus confirmed the successful formation of the MOF structure.

The nineteenth paper, written by M.I. Rizaldi, A. Rahman, Deendarlianto, N.B. Prihantini, and Nasruddin, examines the usage of microbubble aeration in growing susceptible microalgae. In this experiment, the authors use the fluid oscillator to produce microbubbles at a reasonable cost. The fluid oscillator has two types: single loop and double loop. The purpose of this paper was to determine the bubble size produced through these variations of the fluid oscillator. They capture the bubble-size data using a high-speed camera at airflow $6 \mathrm{Lpm}, 9 \mathrm{Lpm}, 12 \mathrm{Lpm}$, and $15 \mathrm{Lpm}$ and utilized $10 \mu \mathrm{m}$ micro-porous shafts as the diffuser. The result of their experiment shows that the single-loop fluid oscillator produces the smallest bubble size with a $6 \mathrm{Lpm}$ airflow.

The twentieth paper, written by E. Kusrini, N. Putra, A. Siswahyu, D. Tristantini, W.W. Prihandini, M.I. Alhamid, Y. Yulizar, and A. Usman, investigates the preparation of $\mathrm{TiO}_{2}$-water nanofluid using titanium dioxide nanoparticles $\left(\mathrm{TiO}_{2} \quad \mathrm{NP}_{\mathrm{S}}\right)$ and cationic surfactant (cetyltrimethylammoniumbromide, CTAB). The effects of the sequence method for the preparation of $\mathrm{TiO}_{2}$-water nanofluid, thermal conductivity, the stability of nanofluid, and temperature distribution were investigated. Some parameters were varied: the sonication times varied between 5, 10, and 30 minutes; $\mathrm{TiO}_{2} \mathrm{NPs}$ were varied 1-8\% for volumetric loading; the concentration of CTAB was varied from $0.005-0.035 \mathrm{wt} . \%$, and the $\mathrm{pH}$ was varied from 8 to 12. The procedure sequences of numbers 2 and 5 showed that the distribution particle size of $\mathrm{TiO}_{2}$ nanoparticles in nanofluid have a narrow range $(190.3-208.7 \mathrm{~nm})$ compared to the other methods $(611-5346 \mathrm{~nm})$. The procedure sequence of number 2 is following demineralized water $(100 \mathrm{~mL}), 8 \%$ volumetric loading of $\mathrm{TiO}_{2} \mathrm{NPs}$, ultrasonication time of $10 \mathrm{~min}$ and CTAB of $3.2 \times 10^{-3} \mathrm{M}$, while the procedure sequence of number 5 is in the respective order of demineralized water $(100 \mathrm{~mL}), 8 \%$ volumetric loading of $\mathrm{TiO}_{2} \mathrm{NPs}$, ultrasonication time of 10 min and $\mathrm{pH}$ at 8 . The CTAB surfactant $(0.029 \mathrm{wt} . \%)$ is more effective for influencing the particle-size distribution in nanofluid than those found in the $\mathrm{pH}$. The experimental results show a maximum of $21 \%$ enhancement of thermal conductivity for $8 \%$ volumetric loading of the $\mathrm{TiO}_{2}$ NPs at $\mathrm{pH} 8$ and a fourfold of critical micelle concentration of 0.029 wt. $\%$ from CTAB. This finding has potential for allowing the preparation of stable $\mathrm{TiO}_{2}$-water nanofluid with short ultrasonic time in ten minutes.

We hope this special edition of IJTech provides useful research findings and insight that lead to new knowledge. We invite you to join us in this journal by sending your research for consideration 
With warmest regards from Jakarta,

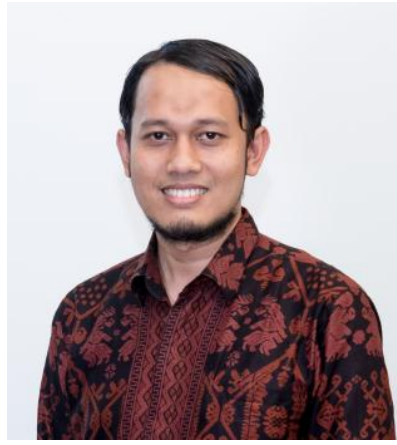

Dr. Muhammad Suryanegara, S.T., M.Sc. Editorial Board Member

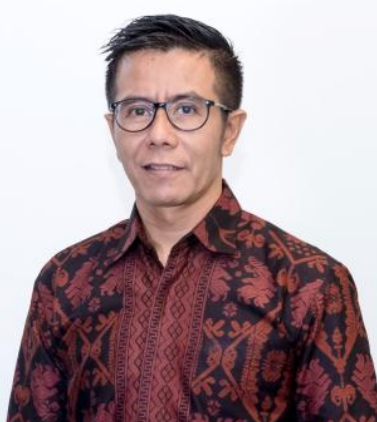

Dr. Ir. Muhamad Asvial, M.Eng. Editorial Board Member

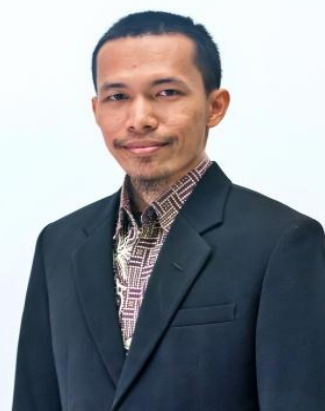

Dr. Ruki Harwahyu, S.T., M.T., M.Sc. Editorial Board Member

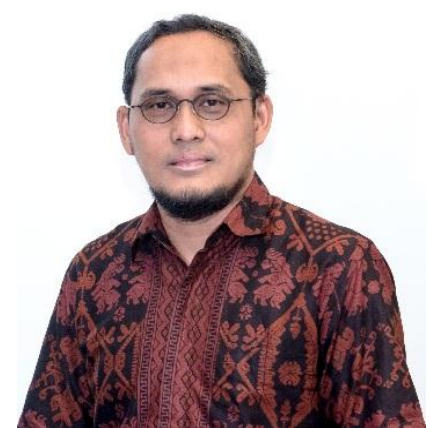

Dr. Ing. Eko Adhi Setiawan, S.T., M.T. Editorial Board Member

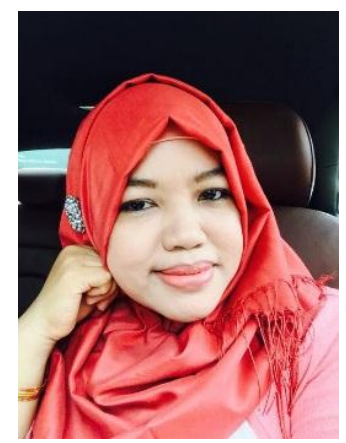

Dr. Eny Kusrini, S.Si.

Editorial Board Member 